Bull. Korean Math. Soc. 44 (2007), No. 3, pp. 517-522

\title{
HYPONORMAL TOEPLITZ OPERATORS ON THE BERGMAN SPACE. II.
}

\author{
In Sung Hwang and Jongrak Lee
}

\begin{abstract}
In this paper we consider the hyponormality of Toeplitz operators $T_{\varphi}$ on the Bergman space $L_{a}^{2}(\mathbb{D})$ with symbol in the case of function $f+\bar{g}$ with polynomials $f$ and $g$. We present some necessary conditions for the hyponormality of $T_{\varphi}$ under certain assumptions about the coefficients of $\varphi$.
\end{abstract}

\section{Introduction}

A bounded linear operator $A$ on a Hilbert space is said to be hyponormal if its selfcommutator $\left[A^{*}, A\right]:=A^{*} A-A A^{*}$ is positive semidefinite. The purpose of this paper is to study hyponormality for Toeplitz operators acting on the Bergman space $L_{a}^{2}(\mathbb{D})$ of the unit disc $\mathbb{D}$. In particular, our interest is Toeplitz operators with polynomial symbols which satisfy certain constraints.

If $P$ denotes the orthogonal projection of $L^{2}(\mathbb{D})$ onto $L_{a}^{2}(\mathbb{D})$, the Toeplitz operator $T_{\varphi}$ on $L_{a}^{2}(\mathbb{D})$ is defined by

$$
T_{\varphi} f=P(\varphi \cdot f)
$$

where $\varphi$ is measurable and $f$ is in $L_{a}^{2}(\mathbb{D})$. It is clear that those operators are bounded if $\varphi$ is in $L^{\infty}(\mathbb{D})$. The Hankel operator $H_{\varphi}: L_{a}^{2} \longrightarrow L_{a}^{2 \perp}$ is defined by $H_{\varphi}(f)=(I-P)(\varphi \cdot f)$. Let $H^{2}(\mathbb{T})$ denote the Hardy space of the unit circle $\mathbb{T}=\partial \mathbb{D}$. Recall that given $\psi \in L^{\infty}(\mathbb{T})$, the Toeplitz operator on $H^{2}(\mathbb{T})$ is the operator $T_{\psi}$ on $H^{2}(\mathbb{T})$ defined by $T_{\psi} f=P_{+}(\psi \cdot f)$, where $f$ is in $H^{2}(\mathbb{T})$ and $P_{+}$denotes the orthogonal projection that maps $L^{2}(\mathbb{T})$ onto $H^{2}(\mathbb{T})$.

Basic properties of the Bergman space and the Hardy space can be found in [1], [3] and [4]. The hyponormality of Toeplitz operators on the Hardy space has been studied by C. Cowen [2], T. Nakazi and K. Takahashi [8], W. Y. Lee [5], [6] and others. In [2], Cowen characterized the hyponormality of Toeplitz operator $T_{\varphi}$ on $H^{2}(\mathbb{T})$ by properties of the symbol $\varphi \in L^{\infty}(\mathbb{T})$. The solution

Received February 10, 2007; Revised April 18, 2007.

2000 Mathematics Subject Classification. Primary 47B20, 47 B35.

Key words and phrases. Toeplitz operators, hyponormal operators, Bergman space. 
is based on a dilation theorem of Sarason [10]. It also exploited the fact that functions in $H^{2 \perp}$ are conjugates of functions in $z H^{2}$. For the Bergman space, $L_{a}^{2 \perp}$ is much larger than the conjugates of functions in $z L_{a}^{2}$, and no dilation theorem (similar to Sarason's theorem) is available. Indeed it is quite difficult to determine the hyponormality of $T_{\varphi}$ on $L_{a}^{2}(\mathbb{D})$. In fact the study of hyponormal Toeplitz operators on the Bergman space seems to be scarce from the literature.

In this paper we study the hyponormality of Toeplitz operators $T_{\varphi}$ on the Bergman space $L_{a}^{2}(\mathbb{D})$ with symbols in the case of function $\varphi=\bar{g}+f$ with polynomials $f$ and $g$. Since the hyponormality of operators is translation invariant we may assume that $f(0)=g(0)=0$. We shall list the well-known properties of Toeplitz operators $T_{\varphi}$ on the Bergman space.

If $f, g$ are in $L^{\infty}(\mathbb{D})$ then we can easily check that

(i) $T_{f+g}=T_{f}+T_{g}$

(ii) $T_{f}{ }^{*}=T_{\bar{f}}$

(iii) $T_{\bar{f}} T_{g}=T_{\bar{f} g}$ if $f$ or $g$ is analytic.

These properties enable us to establish several consequences of hyponormality.

Proposition $1.1([7],[9])$. Let $f, g$ be bounded and analytic. Then the followings are equivalent.

(i) $T_{\bar{g}+f}$ is hyponormal.

(ii) $H_{\bar{g}}^{*} H_{\bar{g}} \leq H_{\bar{f}}^{*} H_{\bar{f}}$.

(iii) $\|(I-P)(\bar{g} k)\| \leq\|(I-P)(\bar{f} k)\|$ for any $k$ in $L_{a}^{2}$.

Very recently, in [7], it was shown that if $\varphi(z)=a_{-m} \bar{z}^{m}+a_{-N} \bar{z}^{N}+a_{m} z^{m}+$ $a_{N} z^{N} \quad(0<m<N)$ and if $a_{m} \overline{a_{N}}=a_{-m} \overline{a_{-N}}$, then

$T_{\varphi}$ is hyponormal

$$
\Longleftrightarrow \begin{cases}\frac{1}{N+1}\left(\left|a_{N}\right|^{2}-\left|a_{-N}\right|^{2}\right) \geq \frac{1}{m+1}\left(\left|a_{-m}\right|^{2}-\left|a_{m}\right|^{2}\right) & \text { if }\left|a_{-N}\right| \leq\left|a_{N}\right| \\ N^{2}\left(\left|a_{-N}\right|^{2}-\left|a_{N}\right|^{2}\right) \leq m^{2}\left(\left|a_{m}\right|^{2}-\left|a_{-m}\right|^{2}\right) & \text { if }\left|a_{N}\right| \leq\left|a_{-N}\right| .\end{cases}
$$

In this paper we continue to examine the hyponormality of $T_{\varphi}$ in the cases where $\varphi$ is a trigonometric polynomial.

\section{Main result}

In this section we present some necessary conditions for hyponormality of $T_{\varphi}$. First of all, observe that for any $s, t$ nonnegative integers,

$$
P\left(\bar{z}^{t} z^{s}\right)= \begin{cases}\frac{s-t+1}{s+1} z^{s-t} & \text { if } s \geq t \\ 0 & \text { if } s<t .\end{cases}
$$


For $0 \leq i \leq N-1$, write

$$
k_{i}(z):=\sum_{n=0}^{\infty} c_{N n+i} z^{N n+i} .
$$

The following lemmas will be used for proving the main result of this section.

Lemma 2.1 ([7]). For $0 \leq m \leq N$, we have

(i) $\left\|\bar{z}^{m} k_{i}(z)\right\|^{2}=\sum_{n=0}^{\infty} \frac{1}{N n+i+m+1}\left|c_{N n+i}\right|^{2}$.

(ii) $\left\|P\left(\bar{z}^{m} k_{i}(z)\right)\right\|^{2}= \begin{cases}\sum_{n=0}^{\infty} \frac{N n+i-m+1}{(N n+i+1)^{2}}\left|c_{N n+i}\right|^{2} & \text { if } m \leq i \\ \sum_{n=1}^{\infty} \frac{N n+i-m+1}{(N n+i+1)^{2}}\left|c_{N n+i}\right|^{2} & \text { if } m>i .\end{cases}$

Lemma 2.2. Let $f(z)=a_{m} z^{m}+a_{N} z^{N}, g(z)=a_{-m} z^{m}+a_{-N} z^{N}(0<m<N)$. If $T_{\bar{g}+f}$ is hyponormal, then

(i) $\frac{1}{N+1}\left(\left|a_{N}\right|^{2}-\left|a_{-N}\right|^{2}\right) \geq \frac{1}{m+1}\left(\left|a_{-m}\right|^{2}-\left|a_{m}\right|^{2}\right)$.

(ii) $\left|a_{m}\right|<\left|a_{-m}\right|$ implies $\left|a_{N}\right|>\left|a_{-N}\right|$.

(iii) $\left|a_{N}\right|<\left|a_{-N}\right|$ implies $\left|a_{m}\right|>\left|a_{-m}\right|$.

Proof. Let $T_{f+\bar{g}}$ be a hyponormal operator. By proposition 1.1, we have $\|f\| \geq$ $\|g\|$. Observe that

$$
\|f\|^{2}=\frac{1}{m+1}\left|a_{m}\right|^{2}+\frac{1}{N+1}\left|a_{N}\right|^{2} \text { and }\|g\|^{2}=\frac{1}{m+1}\left|a_{-m}\right|^{2}+\frac{1}{N+1}\left|a_{-N}\right|^{2} .
$$

This proves the equation (i). The equation (ii) and (iii) are immediate from (i).

Our main result now follows:

Theorem 2.3. Let $\varphi(z)=\overline{g(z)}+f(z)$, where

$$
f(z)=a_{m} z^{m}+a_{N} z^{N} \quad \text { and } \quad g(z)=a_{-m} z^{m}+a_{-N} z^{N} \quad(0<m<N) .
$$

If $T_{\varphi}$ is hyponormal and $\left|a_{N}\right| \leq\left|a_{-N}\right|$, then we have

$$
N^{2}\left(\left|a_{-N}\right|^{2}-\left|a_{N}\right|^{2}\right) \leq m^{2}\left(\left|a_{m}\right|^{2}-\left|a_{-m}\right|^{2}\right)
$$

Proof. Put $k_{i}(z):=\sum_{n=0}^{\infty} c_{N n+i} z^{N n+i}$ for $i=0,1,2, \ldots, N-1$. Then we have

$$
\left\langle k_{i}(z) \bar{z}^{m}, k_{i}(z) \bar{z}^{N}\right\rangle=0 .
$$


Thus by Lemma 2.1, we have

(2)

$$
\begin{aligned}
& \left\langle M_{\bar{f}} k_{i}(z), M_{\bar{f}} k_{i}(z)\right\rangle \\
= & \left|a_{m}\right|^{2} \sum_{n=0}^{\infty} \frac{1}{N n+m+i+1}\left|c_{N n+i}\right|^{2}+\left|a_{N}\right|^{2} \sum_{n=0}^{\infty} \frac{1}{N n+N+i+1}\left|c_{N n+i}\right|^{2}
\end{aligned}
$$

and

(3)

$$
\begin{aligned}
& \left\langle M_{\bar{g}} k_{i}(z), M_{\bar{g}} k_{i}(z)\right\rangle \\
= & \left|a_{-m}\right|^{2} \sum_{n=0}^{\infty} \frac{1}{N n+m+i+1}\left|c_{N n+i}\right|^{2}+\left|a_{-N}\right|^{2} \sum_{n=0}^{\infty} \frac{1}{N n+N+i+1}\left|c_{N n+i}\right|^{2} .
\end{aligned}
$$

If $i \geq m$, it follows from Lemma 2.1 that

(4)

$$
\begin{aligned}
& \left\langle T_{\bar{f}} k_{i}(z), T_{\bar{f}} k_{i}(z)\right\rangle \\
= & \left|a_{m}\right|^{2} \sum_{n=0}^{\infty} \frac{N n+i-m+1}{(N n+i+1)^{2}}\left|c_{N n+i}\right|^{2}+\left|a_{N}\right|^{2} \sum_{n=1}^{\infty} \frac{N n+i-N+1}{(N n+i+1)^{2}}\left|c_{N n+i}\right|^{2}
\end{aligned}
$$

and

(5)

$$
\begin{aligned}
& \left\langle T_{\bar{g}} k_{i}(z), T_{\bar{g}} k_{i}(z)\right\rangle \\
= & \left|a_{-m}\right|^{2} \sum_{n=0}^{\infty} \frac{N n+i-m+1}{(N n+i+1)^{2}}\left|c_{N n+i}\right|^{2}+\left|a_{-N}\right|^{2} \sum_{n=1}^{\infty} \frac{N n+i-N+1}{(N n+i+1)^{2}}\left|c_{N n+i}\right|^{2} .
\end{aligned}
$$

Combining (2) and (4), we see that

$$
\begin{aligned}
& \left\langle H_{\bar{f}}^{*} H_{\bar{f}} k_{i}(z), k_{i}(z)\right\rangle \\
= & \left|a_{m}\right|^{2} \sum_{n=0}^{\infty}\left(\frac{1}{N n+m+i+1}-\frac{N n+i-m+1}{(N n+i+1)^{2}}\right)\left|c_{N n+i}\right|^{2} \\
+ & \left|a_{N}\right|^{2}\left(\frac{1}{N+i+1}\left|c_{i}\right|^{2}+\sum_{n=1}^{\infty}\left(\frac{1}{N n+N+i+1}-\frac{N n+i-N+1}{(N n+i+1)^{2}}\right)\left|c_{N n+i}\right|^{2}\right)
\end{aligned}
$$

Combining (3) and (5), we see that

$$
\begin{aligned}
& \left\langle H_{\bar{g}}^{*} H_{\bar{g}} k_{i}(z), k_{i}(z)\right\rangle \\
= & \left|a_{-m}\right|^{2} \sum_{n=0}^{\infty}\left(\frac{1}{N n+m+i+1}-\frac{N n+i-m+1}{(N n+i+1)^{2}}\right)\left|c_{N n+i}\right|^{2} \\
+ & \left|a_{-N}\right|^{2}\left(\frac{1}{N+i+1}\left|c_{i}\right|^{2}+\sum_{n=1}^{\infty}\left(\frac{1}{N n+N+i+1}-\frac{N n+i-N+1}{(N n+i+1)^{2}}\right)\left|c_{N n+i}\right|^{2}\right)
\end{aligned}
$$


Therefore applying Proposition 1.1 gives that if $T_{\varphi}$ is hyponormal then

$$
\begin{aligned}
& \left\langle\left(H_{f}^{*} H_{\bar{f}}-H_{\bar{g}}^{*} H_{\bar{g}}\right) k_{i}(z), k_{i}(z)\right\rangle \\
= & \left(\left|a_{m}\right|^{2}-\left|a_{-m}\right|^{2}\right) \sum_{n=0}^{\infty}\left(\frac{1}{N n+m+i+1}-\frac{N n+i-m+1}{(N n+i+1)^{2}}\right)\left|c_{N n+i}\right|^{2} \\
+ & \left(\left|a_{N}\right|^{2}-\left|a_{-N}\right|^{2}\right)\left(\frac{1}{N+i+1}\left|c_{i}\right|^{2}+\sum_{n=1}^{\infty}\left(\frac{1}{N n+N+i+1}\right.\right. \\
& \left.\left.-\frac{N n+i-N+1}{(N n+i+1)^{2}}\right)\left|c_{N n+i}\right|^{2}\right)
\end{aligned}
$$

$\geq 0$

If $\left|a_{N}\right| \leq\left|a_{-N}\right|$, it follows from Lemma 2.2 that $\left|a_{m}\right|>\left|a_{-m}\right|$. Define $\xi$ by

$$
\xi(n):=\frac{\frac{1}{N n+m+i+1}-\frac{N n+i-m+1}{(N n+i+1)^{2}}}{\frac{1}{N n+N+i+1}-\frac{N n+i-N+1}{(N n+i+1)^{2}}} \quad(n \geq 1) .
$$

Then $\xi$ is a strictly decreasing function and

$$
\lim _{n \rightarrow \infty} \xi(n)=\frac{m^{2}}{N^{2}}
$$

Since $\xi(n) \geq \frac{m^{2}}{N^{2}}$, it follows from (6) that $T_{\varphi}$ is hyponormal, then we have

$$
N^{2}\left(\left|a_{-N}\right|^{2}-\left|a_{N}\right|^{2}\right) \leq m^{2}\left(\left|a_{m}\right|^{2}-\left|a_{-m}\right|^{2}\right) .
$$

This completes the proof.

The following example shows that the converse of Theorem 3.2 is not true.

Example. Consider the trigonometric polynomial

$$
\varphi(z)=2 \bar{z}^{2}+2 \bar{z}-4 z+z^{2}
$$

Then $\varphi$ satisfies the inequality (1). But a straightforward calculation shows that

$$
\left\langle\left(H_{\bar{f}}^{*} H_{\bar{f}}-H_{\bar{g}}^{*} H_{\bar{g}}\right)(1+2 z),(1+2 z)\right\rangle=9 \frac{1}{3}-14<0 .
$$

Therefore $T_{\varphi}$ is not hyponormal.

\section{References}

[1] S. Axler, Bergman spaces and their operators, Surveys of some recent results in operator theory, Vol. I, 1-50, Pitman Res. Notes Math. Ser., 171, Longman Sci. Tech., Harlow, 1988.

[2] C. Cowen, Hyponormality of Toeplitz operators, Proc. Amer. Math. Soc. 103 (1988), no. $3,809-812$.

[3] R. Douglas, Banach algebra techniques in operator theory, Pure and Applied Mathematics, Vol. 49. Academic Press, New York-London, 1972.

[4] P. Duren, Theory of $H^{p}$ spaces, Pure and Applied Mathematics, Vol. 38 Academic Press, New York-London, 1970. 
[5] I. S. Hwang, I. H. Kim, and W. Y. Lee, Hyponormality of Toeplitz operators with polynomial symbols, Math. Ann. 313 (1999), no. 2, 247-261.

[6] I. S. Hwang and W. Y. Lee, Hyponormality of trigonometric Toeplitz operators, Trans. Amer. Math. Soc. 354 (2002), no. 6, 2461-2474.

[7] I. S. Hwang, Hyponormal Toeplitz operators on the Bergman space, J. Korean Math. Soc. 42 (2005), no. 2, 387-403.

[8] T. Nakazi and K. Takahashi, Hyponormal Toeplitz operators and extremal problems of Hardy spaces, Trans. Amer. Math. Soc. 338 (1993), no. 2, 753-767.

[9] H. Sadraoui, Hyponormality of Toeplitz operators and Composition operators, Thesis, Purdue University, 1992.

[10] D. Sarason, Generalized interpolation in $H^{\infty}$, Trans. Amer. Math. Soc. 127 (1967), 179-203.

In Sung HWang

Department of Mathematics

SUNGKYUNKWAN UNIVERSITY

SUWON 440-746, KOREA

E-mail address: ihwang@skku.edu

JONGRAK LEE

Department of Mathematics

SUNGKYUNKWAN UNIVERSITY

Suwon 440-746, Korea

E-mail address: jjonglak@skku.ac.kr 